\title{
Non-Invasive Risk Stratification in NAFLD/NASH Patients for Screening EGD
}

\author{
John Romano' \\ Thaer Abdelfattah ${ }^{2}$ \\ Paul P Manka ${ }^{3}$ \\ Michael Fuchs ${ }^{2,4}$ \\ Wing-Kin Syn ${ }^{1,5,6}$ \\ 'Division of Gastroenterology and \\ Hepatology, Medical University of South \\ Carolina, Charleston, SC, USA; ${ }^{2}$ Division \\ of Gastroenterology and Hepatology, \\ Central Virginia VA Health Care System, \\ Richmond, VA, USA; ${ }^{3}$ Department of \\ Internal Medicine, University Hospital, \\ Knappschaftskrankenhaus, Ruhr- \\ University Bochum, Bochum, Germany; \\ ${ }^{4}$ Division of Gastroenterology and \\ Hepatology, Virginia Commonwealth \\ University, Richmond, VA, USA; ${ }^{5}$ Section \\ of Gastroenterology, Ralph $\mathrm{H}$ Johnson \\ VAMC, Charleston, SC, USA; \\ ${ }^{6}$ Department of Physiology, Faculty of \\ Medicine and Nursing, University of the \\ Basque Country, Universidad del Pa S \\ Vasco/Euskal Herriko Univertsitatea \\ (UPV/EHU), Leioa, Spain
}

Correspondence: John Romano Division of Gastroenterology and Hepatology, Medical University of South Carolina, Strom Thurmond Gazes Cardiac Research Institute 30 Courtenay Drive Suite: 249, Charleston, SC, USA Email Romanoj@musc.edu
Portal hypertension is a major complication of cirrhosis, as it predisposes patients to manifestations of hepatic decompensation, including the development of esophageal variceal bleeding, hepatic encephalopathy, and ascites. ${ }^{1}$ Esophageal varices are present in approximately $50 \%$ of patients with cirrhosis. The mortality during a variceal bleeding event is high, with estimates ranging from $15 \%$ to $20 \%$, being largely dependent upon whether patients have received the standard of care with endoscopic band ligation, vasoactive drugs, and antibiotics. ${ }^{2}$ The hepatic venous pressure gradient (HVPG) is considered the gold standard in ascertaining the presence of portal hypertension $(\mathrm{PH})$. Clinically significant portal hypertension (CSPH), which is associated with the development of the aforementioned manifestations of decompensation, has been defined as an HVPG greater than or equal to $10 \mathrm{mmHg}$. After a diagnosis of cirrhosis, current guidelines recommend screening for esophageal varices with esophagogastroduodenoscopy (EGD). This procedure is carried out to identify patients who are at risk for variceal hemorrhage and would benefit from starting prophylactic therapy with beta blockade. ${ }^{3,4}$ This procedure carries risks, which include any type of respiratory or cardiac suppression from anesthesia, infection, bleeding, and perforation. In addition, a significant majority of patients with varices who are indeed at high risk for bleeding do not have any symptoms from the varices themselves, making EGD a non-ideal screening test. ${ }^{5}$ Previous studies suggest that non-invasive blood-based markers are useful to identify patients with liver damage (fibrosis or cirrhosis) and may identify those who will develop complications such as liver cancer. ${ }^{6}$ Examples of these markers include the Fibrosis-4 Index (FIB-4), which is a non-invasive estimate of liver scarring in HCV and HBV patients; the NAFLD (Non-Alcoholic Fatty Liver Disease) Fibrosis Score (NFS), which is used to estimate the amount of scarring in the liver based on several laboratory tests; the BARD score, based upon BMI, AST/ALT ratio, and the presence or absence of diabetes; and the AST to Platelet Ratio Index (APRI). We evaluated whether these non-invasive markers and/or any other clinical parameters may be used to identify patients with liver cirrhosis who are likely to have large esophageal varices, and therefore would benefit most from screening EGD.

We retrospectively evaluated a cohort of non-alcoholic fatty liver disease (NAFLD)/non-alcoholic steatohepatitis (NASH) patients at two tertiary care Veterans Affairs (VA) Hospitals between January 1st 2017 and February 15th 2021. A total of 1476 patients was initially investigated; however, 1221 patients 
were excluded owing to stage 1 or 2 fibrosis on fibroscan or liver biopsy. Patients who had advanced liver fibrosis or cirrhosis on the basis of elastography measurements and/or liver biopsies were further evaluated. Endoscopic records for these patients were reviewed for the presence and size of esophageal varices. Variables that were collected included race, sex, BMI, age, liver enzymes (including AST/ALT), platelet count, albumin, uric acid levels, and the presence or absence of comorbidities, including diabetes, to calculate the BARD score, FIB-4, APRI, and NFS. We also evaluated the utility of the BAVENO VI expanded criteria (patients with liver stiffness $<20 \mathrm{kPa}$ and platelet count $>150,000 / \mu \mathrm{L}$ are at low risk of having varices that require treatment and, therefore, do not require screening EGD) in this cohort. ${ }^{7}$

Forty-four patients met the inclusion criterion, which is defined as stage 3 or 4 fibrosis or cirrhosis on the basis of elastography and/or liver biopsies. These patients were divided into two groups. Group A: 26 subjects with small esophageal varices or no varices (mean age: 59.3 years; male: 24, female: 2; African American: 2, Asian: 3, White: 19); group B: 18 subjects with large esophageal varices (mean age: 65.4 years; male: 16, female: 2; African American: 1, Asian: 2, White: 15). Patients in group B had significantly higher non-invasive scores than those in group A: FIB-4 (1.87 vs 3.28; $p<0.001)$, APRI (0.39 vs $1.08 ; p<0.001)$, NFS ( 0.58 vs $1.82 ; p<0.01)$, and BARD ( 2 vs $3 ; p<0.05)$. FIB-4 provided the overall highest AUROC of 0.82 (specificity: 77\%; NPV: $74 \%$ ) for the presence of large varices. While APRI showed an AUROC of 0.812 (specificity: 100\%; NPV: 70\%), NFS had an AUROC of 0.777 (specificity: 54\%; NPV: 88\%), and BARD presented an AUROC of 0.696 (specificity: 65\%; NPV: 73\%). In addition, BAVENO VI expanded criteria were able to significantly distinguish between groups A and B by chi-squared analysis $(p=0.0139)$ and Fisher's exact test $(p=0.0292)$. We also found significant differences between groups A and B with regard to the presence of splenomegaly $(p=0.046)$ and uric acid levels $(p=0.027)$. Non-invasive tests are increasingly being used to stratify the risks of patients with chronic liver disease. As these tests have excellent negative predictive value, they are generally used to identify those who are unlikely to have significant or advanced liver disease, and therefore do not require invasive evaluation such as a liver biopsy. This study extends their utility and suggests that the FIB-4 and APRI may be able to accurately identify individuals likely to have large esophageal varices and who would therefore benefit most from screening EGD. The extended BAVENO VI guidelines further recommend that patients with liver stiffness $<15 \mathrm{kPa}$ and platelet count $>150,000 / \mu \mathrm{L}$ can safely avoid endoscopic screening for varices owing to a very low probability of their having large varices that require treatment. ${ }^{7}$ Petta et al retrospectively investigated a large cohort and showed that $58 \%$ of EGDs could be spared using the extended BAVENO VI criteria, while missing only $0.9 \%$ of varices that would need treatment. ${ }^{8}$ Our data build upon this information. Additional parameters that should be investigated to assess for the presence or absence of varices include splenomegaly and uric acid levels, as there was a significant difference between groups $\mathrm{A}$ and $\mathrm{B}$ for both of these factors. Findings from a 2021 study suggest that there is a relationship between splenomegaly and NAFLD/NASH. ${ }^{9}$ Spleen stiffness on elastography is also a developing non-invasive indicator for the presence or absence of esophageal varices and should be studied further.

Overall, this VA study is clinically significant because it may allow providers to better identify individuals with advanced liver disease who are most at risk for variceal bleed and who need a screening EGD. Conversely, those with low non-invasive scores, FIB- $4<1.45$ or APRI $<0.5$, or who do not meet BAVENO VI criteria, may be able to avoid unnecessary EGDs. Further prospective studies with larger cohorts will be needed to validate these findings.

\section{Ethics and Consent}

This study was approved by the institutional review board at the Ralph H Johnson VAMC. Patient consent was not required to review medical records and this was not completed owing to the time frame of the study (many years and some patient deaths unrelated to the study). Patient consent was obtained for any necessary procedure or intervention that was included in the study. The consent was obtained at the time the original study was completed. Patients were deidentified to ensure that no patient information was exposed, in compliance with the Declaration of Helsinki.

\section{Disclosure}

The authors report no conflicts of interest for this work.

\section{References}

1. de Franchis R Revising consensus in portal hypertension: report of the Baveno $\mathrm{V}$ consensus workshop on methodology of diagnosis and therapy in portal hypertension. J Hepatol. 2010;53:762-768 
2. Augustin S, Altamirano J, González A, et al. Effectiveness of combined pharmacologic and ligation therapy in high risk patients with acute esophageal variceal bleeding. Am $J$ Gastroenterol. 2011;106:1787-1795. doi:10.1038/ajg.2011.173

3. Garcia-Tsao G, Abraldes JG, Berzigotti A, et al. Portal hypertensive bleeding in cirrhosis: risk stratification, diagnosis, and management: 2016 practice guidance by the American Association for the study of liver diseases. Hepatology. 2017;65:310. doi:10.1002/hep.28906

4. de Franchis R Expanding consensus in portal hypertension: report of the Baveno VI Consensus Workshop: stratifying risk and individualizing care for portal hypertension. J Hepatol. 2015;63:743-752

5. Karagiannakis DS, Voulgaris T, Koureta E, et al. Role of Spleen Stiffness Measurement by 2D-Shear Wave Elastography in Ruling Out the Presence of High-Risk Varices in Cirrhotic Patients. Digestive Dis Sci. 2019;64:2653-2660. doi:10.1007/s10620-01905616-4
6. D’Amico G, Garcia-Tsao G, Pagliaro L. Natural history and prognostic indicators of survival in cirrhosis: a systematic review of 118 studies. J Hepatol. 2006;44:217-231. doi:10.1016/j.jhep.2005.10.013

7. Augustin S, Pons M, Maurice JB, et al. Expanding the Baveno VI criteria for the screening of varices in patients with compensated advanced chronic liver disease. Hepatology. 2017;66(6):1980-1988. doi:10.1002/hep.29363

8. Petta S, Sebastiani G, Bugianesi E, et al. Non-invasive prediction of esophageal varices by stiffness and platelet in non-alcoholic fatty liver disease cirrhosis. J Hepatol. 2018;69(4):878-885. doi:10.1016/ j.jhep.2018.05.019

9. Tarantion G, Citro V, Balsano C. Liver-spleen axis in nonalcoholic fatty liver disease. Exp Rev Gastroenterol Hepatol. 2021;7:759-769. doi:10.1080/17474124.2021.1914587

\section{Publish your work in this journal}

Clinical and Experimental Gastroenterology is an international, peerreviewed, open access, online journal publishing original research, reports, editorials, reviews and commentaries on all aspects of gastroenterology in the clinic and laboratory. This journal is indexed on American Chemical Society's Chemical Abstracts Service (CAS)
The manuscript management system is completely online and includes a very quick and fair peer-review system, which is all easy to use. Visit http://www.dovepress.com/testimonials.php to read real quotes from published authors. 